\title{
Concurrent Composition in the Bounded Quantum Storage Model
}

\author{
Dominique Unruh
}

Saarland University

\begin{abstract}
We define the BQS-UC model, a variant of the UC model, that deals with protocols in the bounded quantum storage model. We present a statistically secure commitment protocol in the BQS-UC model that composes concurrently with other protocols and an (a-priori) polynomially-bounded number of instances of itself. Our protocol has an efficient simulator which is important if one wishes to compose our protocol with protocols that are only computationally secure. Combining our result with prior results, we get a statistically BQS-UC secure constant-round protocol for general two-party computation without the need for any setup assumption.
\end{abstract}

Keywords: Bounded quantum storage, composability, two-party computation.

\section{Introduction}

Since the inception of quantum key distribution by Bennett and Brassard [2], it has been known that quantum communication permits to achieve protocol tasks that are impossible given only a classical channel. For example, a quantum key distribution scheme [2] permits to agree on a secret key that is statistically secret, using only an authenticated but not secret channel. (By statistical security we mean security against computationally unbounded adversaries, also known as information-theoretical security.) In contrast, when using only classical communication, it is easy to see that such a secret key can always be extracted by a computationally sufficiently powerful adversary. In light of this result, one might hope that quantum cryptography allows to circumvent other classical impossibility results, possibly even allowing for statistically secure multi-party computation protocols. Yet, Mayers [13] showed that also in the quantum setting, even statistically secure commitment schemes are impossible, let alone general multi-party computation. This is unfortunate, because from commitments one can build OT (Bennett, Brassard, Crépeau, and Skubiszewska [3]), and from OT general multiparty computation (Kilian [1] ). A way to work around this impossibility was found by Damgård, Fehr, Salvail, and Schaffner [6]. They showed that if we assume that the quantum memory available to the adversary is bounded (we speak of bounded quantum storage (BQS)), we can construct statistically secure commitment and OT schemes. Although such a result is not truly unconditional, it avoids hard-to-justify complexity-theoretic assumptions. Also, it achieves longterm security: even if the adversary can surpass the memory bound after the protocol execution, this will not allow him to retroactively break the protocol.

K.G. Paterson (Ed.): Eurocrypt 2011, LNCS 6632, pp. 467-486, 2011.

(C) International Association for Cryptologic Research 2011 
Yet, we still have not reached the goal of statistically secure multi-party computation. Although we have protocols for commitment and OT, we cannot simply plug them into the protocols by Bennett et al. 3] and by Kilian [11. The reason is that it is not clear under which circumstances protocols in the BQS model may be composed. For example, Dziembowski and Maurer [7] constructed a protocol that is secure in the classical bounded storage model, but that looses security when composed with a computationally secure protocol. To overcome this remaining difficulty, works by Wehner and Wullschleger [17] and by Fehr and Schaffner [8] give security definitions in the BQS model that enable secure sequential composition. Both works also present secure OT protocols in their respective settings. Based on these, we can construct secure multi-party computation protocols in the BQS model. There are, however, a few limitations. First, since only sequential composition is supported, all instances of the OT protocol used by the multi-party computation need to be executed one after another, leading to a high round-complexity. Second, interactive functionalities such as a commitment are difficult to use: the restriction to sequential composability requires that we have to commit and immediately open a commitment before being allowed to execute the next commitment. Third, the security proof of their OT protocols uses a computationally unlimited simulator. As discussed in [15, a protocol with an unlimited simulator cannot be composed with a computationally secure protocol. Fourth, since we have no concurrent composability, it is not clear what happens if the protocols are executed in an environment where we do not have total control about which protocols are executed at what time.

To overcome the limitations of sequential composition in the classical setting, Canetti [4] introduced the Universal Composability (UC) model. In this model, protocols can be arbitrarily composed, even concurrently with other protocols and with copies of themselves. The UC model has been adapted to the quantum setting by Ben-Or and Mayers [1] and by Unruh [1415]. In light of the success of the UC model, it seems natural to combine the ideas of the UC model with those of the BQS model in order to allow for concurrent composition.

\subsection{Our Contribution}

We define the notion of BQS-UC-security, which is an extension of quantumUC-security [15. We have composability in the following sense: If $\pi$ is a secure realization of a functionality $\mathcal{F}$, and $\sigma^{\mathcal{F}}$ securely realizes $\mathcal{G}$ by using one instance of $\mathcal{F}$, then $\sigma^{\pi}$, the result of replacing $\mathcal{F}$ by $\pi$, still securely realizes $\mathcal{G}$. In contrast to quantum-UC-security, however, BQS-UC-security does not allow for concurrent self-composition: if $\pi$ is secure, this does not automatically imply that two concurrent instances of $\pi$ are secure 1 .

\footnotetext{
${ }^{1}$ The reader may wonder how it can be that $\sigma$ and $\pi$ may compose in general while $\pi$ and $\pi$ do not. Might not $\sigma$ and $\pi$ be the same protocol? The reason lies in the exact conditions of the composition theorem: In order to compose, $\sigma$ needs to be secure against adversaries with a higher quantum memory bound than $\pi$ tolerates. Thus $\sigma$ and $\pi$ cannot be the same protocol.
} 
In order to get protocols that even self-compose concurrently, we design a commitment scheme $\pi_{\mathrm{COM}}$ such that $n$ concurrent instances of $\pi_{\mathrm{COM}}$ securely realize $n$ instances of the commitment functionality in the presence of $a$-memory bounded adversaries. Here $a$ and $n$ are arbitrary (polynomially-bounded), but the protocol depends on $a$ and $n$.

The challenging part in the construction of $\pi_{\mathrm{COM}}$ is that BQS-UC-security requires the following: There must be an efficient simulator (which is allowed to have more quantum memory than the adversary) that can extract the committed value (extractability) or change it after the commit phase (equivocality). Prior constructions of commitment schemes in the BQS model required computationally unbounded simulators. Also, the fact that we directly analyze the concurrent composition of several instances of $\pi_{\mathrm{COM}}$ requires care: In the proof, we have hybrid networks in which instances of both $\pi_{\mathrm{COM}}$ and of the simulator occur. Since the simulator uses more quantum memory than $\pi_{\mathrm{COM}}$ tolerates, one needs to ensure that the simulator cannot be (mis) used by the adversary to break the commitment.

Finally, using the composition theorem and $\pi_{\mathrm{COM}}$, for any two-party functionality $\mathcal{G}$, we get a statistically secure protocol $\pi$ realizing $\mathcal{G}$ in the BQS mode 2 . The protocol is secure even when running $n$ concurrent instances of the protocol. (Again, this holds for any $n$ and any memory bound, but the protocol depends on $n$ and the memory-bound.) The protocol is constant-round. It does not use any quantum memory or quantum computation and thus is in the reach of today's technology.

A full version of this paper with complete proofs and details can be found at 16 .

\section{Bounded Quantum Storage UC}

\subsection{The BQS-UC Model}

To understand the definition of BQS-UC-security, we first have to understand the idea underlying UC security. In the UC model, a protocol $\pi$ emulates (realizes, implements, is as secure as) another protocol $\rho$ if any attack on $\pi$ can also happen on $\rho$. Thus, if $\rho$ is secure by definition (e.g., because it contains only one trusted machine, a so-called ideal functionality), $\pi$ must also be secure. To formalize this, we introduce the concept of an adversary and a simulator. We require that for any adversary attacking $\pi$ (real model), there is a simulator attacking $\rho$ (ideal model) such that the real and the ideal model are indistinguishable. To define indistinguishability, we introduce another machine, the environment. Its task is to try and distinguish between the real and the ideal model. The environment provides the inputs to the protocol parties, gets their outputs, and may talk to the adversary/simulator. We then get the following definition: $\pi$ UC-emulates $\rho$

\footnotetext{
${ }^{2}$ We are restricted to two-party functionalities because our construction uses a subprotocol by Wolf and Wullschleger [18,19] to reverse the direction of an OT; this protocol only makes sense in a two-party setting.
} 
if for any adversary Adv there is a simulator Sim such that for all environments $\mathcal{Z}$, the probability that $\mathcal{Z}$ outputs 1 is approximately the same in the networks $\pi, \operatorname{Adv}, \mathcal{Z}$ and $\rho, \operatorname{Sim}, \mathcal{Z}$.

To translate this to the quantum setting, we only need to change the machine model to allow for quantum machines instead of classical machines. Given networks $S, S^{\prime}$ of quantum machines with $\mathcal{Z} \in S, S^{\prime}$, we say that $S$ and $S^{\prime}$ are $\varepsilon$-close if $\left|P-P^{\prime}\right| \leq \varepsilon$ where $P$ is the probability that $\mathcal{Z}$ outputs 1 in an execution of $S$, and $P^{\prime}$ is defined analogously. Networks are negligible-close if $\varepsilon$ is negligible, and perfectly close if $\varepsilon=0$. We call a machine $M a$-memory bounded if it keeps at most $a$ qubits of quantum memory between activations. (An activation is the computation performed by a machine between receiving a message and sending the immediate response.) We do not impose any limitations on the computationtime or memory-use during a single activation of $M$. We write $\mathrm{QM}(M)$ for the memory bound of $M$ (i.e., $\mathrm{QM}(M)$ is the smallest $a$ such that $M$ is $a$-memory bounded). A protocol $\pi$ is a network not containing adversary, simulator, or environment. The set of corruptible protocol parties is denoted parties $_{\pi}$. Given $C \subseteq$ parties $_{\pi}$, we denote by $\pi^{C}$ the protocol where all parties in $C$ have been replaced by corruption parties which are controlled by the adversary. For details on the machine and the network model, we refer to the full version [16] or to [15].

We can now formulate BQS-UC-security. Intuitively, a protocol is BQS-UCsecure if it is UC-secure for memory-bounded adversaries. To formulate this, we need to explicitly parametrize the definition over a memory bound $a$. Then we require that the total quantum memory used by environment and adversary is bounded by $a$. The reason why we include the environment's memory is that the latter can be involved in the actual attack: If only the adversary's memory was bounded, the adversary could use the environment as an external storage to perform the attack (see also our discussion on page 471) 3 .

It remains to decide whether the simulator should be memory bounded. If we allow the simulator to be unbounded, composition becomes difficult: In some cases, the simulator of one protocol plays the role of the adversary of a second protocol. Thus, if simulators where not memory bounded, the second protocol would have to be secure against unbounded adversaries. However, if we require the simulator to be $a$-memory bounded, we will not be able to construct nontrivial protocols: In order to perform a simulation, the simulator needs to have some advantage over an honest protocol participant (in the computational UC setting, e.g., this is usually the knowledge of some trapdoor). In our setting, the advantage of the simulator will be that he has more quantum memory than the adversary. Thus we introduce a second parameter $s$ which specifies the amount of quantum memory the simulator may use for the simulation. More precisely, we allow the simulator to use $s+\mathrm{QM}(\mathrm{Adv})$ qubits because the simulator will usually internally simulate the adversary Adv as a black-box and therefore have to additionally reserve sufficient quantum memory to store the adversary's state.

${ }^{3}$ This is captured more formally by the completeness of the so-called dummyadversary (see the full version [16]), which shows that one can even shift the complete attack into the environment. 
Definition 1 (BQS-UC-security). Fix protocols $\pi$ and $\rho$. Let $a, s \in \mathbb{N}_{0} \cup$ $\{\infty\}$ (possibly depending on the security parameter). We say $\pi(a, s)$-BQS-UCemulates 4 iff for every set $C \subseteq$ parties $_{\pi}$ and for every adversary Adv there is a simulator $\operatorname{Sim}$ with $\mathrm{QM}(\mathrm{Sim}) \leq s+\mathrm{QM}(\mathrm{Adv})$ such that for every environment $\mathcal{Z}$ with $\mathrm{QM}(\mathcal{Z})+\mathrm{QM}(\mathrm{Adv}) \leq a$, the networks $\pi^{C} \cup\{\mathrm{Adv}, \mathcal{Z}\}$ (called the real model) and $\rho^{C} \cup\{\operatorname{Sim}, \mathcal{Z}\}$ (called the ideal model) are negligible-close. We furthermore require that if $\mathrm{Adv}$ is quantum-polynomial-time, so is Sim.

In most cases, the behavior of the ideal protocol $\rho$ is described by a single machine $\mathcal{F}$, the so-called ideal functionality. We can think of this functionality as a trusted third party that perfectly implements the desired protocol behavior. In order to apply Definition 1 to ideal functionalities (e.g., $\pi$ BQS-UC-emulates $\mathcal{F}$ ) we have to be able to consider an ideal functionality as a protocol. Following 415], we do this by introducing dummy-parties. That is, the protocol $\mathcal{F}$ consists of the functionality $\mathcal{F}$ together with a dummy-party $\tilde{A}$ for every party $A$. The dummy-parties just forward inputs/outputs between the functionality $\mathcal{F}$ and the environment. They can, however, be corrupted by the adversary/simulator. This allows the adversary/simulator to control the inputs/outputs of that party. When we write $\pi$ BQS-UC-emulates $\mathcal{F}$, we always assume the presence of dummyparties in the ideal model. For details, we refer to the full version [16] or to [15].

On the Memory Bound of the Environment. In Definition 1, we impose the memory bound on both the adversary and the environment. In this, we differ from the modeling by Wehner and Wullschleger [17. In their definition, the environment (which is implicit in the definition of the indistinguishability $\equiv_{\varepsilon}$ of quantum channels) provides the input state to protocol and adversary, then gets the outputs of protocol and adversary, and finally the environment has to guess whether it interacted with the real or the ideal model. During the interaction of the protocol, the environment is not allowed to communicate with any other machine. Between its two activations, the environment is allowed to keep an arbitrarily large quantum state5. The interesting point here is that, in contrast to our Definition 1, Wehner and Wullschleger do not impose the memory bound on the environment, only on the adversary. They motivate unlimited environments by pointing out that it is more realistic to assume that a particular memory bound (say, 100 qubits) applies to a particular adversary (e.g., a smart card) than to the whole environment (i.e., all computers world-wide). We believe, however, that this reasoning has to be applied with care: Only when we have

${ }^{4}$ Since we only consider statistical security in this work, we omit the qualifier "statistical". Similarly, when we speak about classical-UC-security and quantum-UC-security, we mean the statistical variant of that notion.

${ }^{5}$ Note that strictly speaking, the formalism of [17, full version] does not model an environment with quantum memory: For quantum channels $\Lambda, \Lambda^{\prime}$ they define $\Lambda \equiv_{\varepsilon} \Lambda^{\prime}$ iff for all quantum states $\rho$, the trace distance between $\Lambda(\rho)$ and $\Lambda^{\prime}(\rho)$ is at most $\varepsilon$. To model environments with quantum memory, we should instead require that for all Hilbert spaces $\mathcal{H}$ and all quantum states $\rho$, the trace distance between $\left(\Lambda \otimes i d_{\mathcal{H}}\right)(\rho)$ and $\left(\Lambda^{\prime} \otimes i d_{\mathcal{H}}\right)(\rho)$ is at most $\varepsilon$. We believe that the latter was the intended meaning of $\equiv_{\varepsilon}$. 
the guarantee that the adversary (e.g., the smart card) cannot communicate with any other machines can we assume a smart card is limited to 100 qubits. Otherwise, we have to assume that the smart card effectively has (in the worst case) access to all the quantum memory of the environment. Thus, except in very specific cases, the memory bound we assume needs to be large enough to encompass the environment's memory as a whole. Thus, the bound we assume not to be surpassed by the adversary's memory needs to be large enough that it makes sense to assume that the environment does not surpass this bound either. But in this case, we can safely assume in Definition 1 that the environment is restricted by that memory bound.

We stress that even if our environment is memory bounded, we do take into account the fact that an environment can have a quantum state that is entangled with that of the adversary; we just limit this quantum state to the memory bound.

We get, however, an interesting variant of our model if we follow the approach of Wehner and Wullschleger as follows. We call a machine $a-\diamond$-memory-bounded 6 if its state between activations consists of two registers $A$ and $B$. The register $A$ contains at most $a$ qubits, and register $B$ is unlimited but is only accessed in the first and the last activation of $B$. We denote by $\mathrm{QM}_{\diamond}(\mathcal{Z})$ the $\diamond$-memory bound of $\mathcal{Z}$. We define $(a, s)$ - $\diamond$-BQS-UC-emulation like $(a, s)$-BQS-UC-emulation (Definition 1), except that we use $\mathrm{QM}_{\diamond}(\mathcal{Z})$ instead of $\mathrm{QM}(\mathcal{Z})$. (But we still use $\mathrm{QM}(\mathrm{Adv})$ and $\mathrm{QM}(\mathrm{Sim})$.) We stress that our techniques also work for this definition. All results of this section still hold with essentially unmodified proofs (except that we always have to refer to the $\diamond$-memory bound of the environment instead memory bound). The results from Section 3 (BQS-UC commitments) are based on the existence of certain commitment schemes that are hiding with respect to memory bounded adversaries. We use the commitment scheme from [12] (see Theorem 6). To extend the results from Section 3 to $\diamond$-BQS-UC, we need schemes that are hiding with respect to $\diamond$-memory bounded adversaries instead. Besides that, the proofs of the results in Section 3 stay essentially the same (except for using $\diamond$-memory bounds instead of memory bounds).

\subsection{Composition}

For some protocol $\sigma$, and some protocol $\pi$, by $\sigma^{\pi}$ we denote the protocol where $\sigma$ invokes (up to polynomially many) instances of $\pi$. That is, in $\sigma^{\pi}$ the machines from $\sigma$ and from $\pi$ run together in one network, and the machines from $\sigma$ access the inputs and outputs of $\pi$. (That is, $\sigma$ plays the role of the environment from the point of view of $\pi$. In particular, $\mathcal{Z}$ then talks only to $\sigma$ and not to the subprotocol $\pi$ directly.) A typical situation would be that $\sigma^{\mathcal{F}}$ is some protocol that makes use of some ideal functionality $\mathcal{F}$, say a commitment functionality, and then $\sigma^{\pi}$ would be the protocol resulting from implementing that functionality with some protocol $\pi$, say a commitment protocol. One would hope that such an implementation results in a secure protocol $\sigma^{\pi}$. That is, we hope that if $\pi$

\footnotetext{
${ }^{6}$ We use the symbol $\diamond$ because $\diamond$-memory-bounded environments essentially model indistinguishability with respect to the so-called $\diamond$-norm.
} 
BQS-UC-emulates $\mathcal{F}$ and $\sigma^{\mathcal{F}}$ BQS-UC-emulates $\mathcal{G}$, then $\sigma^{\pi}$ BQS-UC-emulates $\mathcal{G}$. Fortunately, this is the case, as long as we pick the memory bounds in the right way:

Theorem 2 (Composition Theorem). Let $\pi$ and $\sigma$ be quantum-polynomialtime protocols and $\mathcal{F}$ and $\mathcal{G}$ be quantum-polynomial-time functionalities. Assume that $\sigma$ invokes at most one subprotocol instance. Assume that $\pi(a, s)-B Q S-U C$ emulates $\mathcal{F}$ and that $\sigma^{\mathcal{F}}\left(a-\mathrm{QM}(\sigma)+s, s^{\prime}\right)$-BQS-UC-emulates $\mathcal{G}$. Then $\sigma^{\pi}$ $\left(a-\mathrm{QM}(\sigma), s+s^{\prime}\right)$-BQS-UC-emulates $\mathcal{G}$.

The proof of this theorem is very similar to that in [15], except that we have to keep track of the quantum memory used by various machines constructed in the proof.

Notice that in this composition theorem, the outer protocol $\sigma$ is only allowed to invoke one instance of the subprotocol $\pi$. This stands in contrast to the universal composition theorem for classical-UC [4] and for quantum-UC [15] where any polynomially-bounded number of concurrent instances of $\pi$ is allowed. In fact, this is not just a limitation of our proof technique7. For example, assume a protocol $\pi_{\mathrm{COM}}^{A \rightarrow B}$ that $(a, s)$-BQS-UC-emulates the commitment functionality $\mathcal{F}_{\mathrm{COM}}^{A \rightarrow B}$ with sender $A$ and recipient $B$. Assume further that $\pi_{\mathrm{COM}}^{A \rightarrow B}$ does not use any functionalities as setup. As we will see later, such a protocol exists. Now let $\pi_{\mathrm{COM}}^{B \rightarrow A}$ be the protocol that results from exchanging the roles of $A$ and $B$. Then $\pi_{\mathrm{COM}}^{B \rightarrow A}(a, s)$-BQS-UC-emulates $\mathcal{F}_{\mathrm{COM}}^{B \rightarrow A}$. Consider the concurrent composition of $\pi_{\mathrm{COM}}^{A \rightarrow B}$ and $\pi_{\mathrm{COM}}^{B \rightarrow A}$. In this protocol, a corrupted Bob may reroute all messages between the Alice in the first protocol and Alice in the second protocol. Thus, if Alice commits to a random value $v$ in the first protocol, Bob commits to the same value $v$ in the second protocol without knowing it. It is easy to see that in a concurrent composition of $\mathcal{F}_{\mathrm{COM}}^{A \rightarrow B}$ and $\mathcal{F}_{\mathrm{COM}}^{B \rightarrow A}$, this is not possible. Thus the composition of $\pi_{\mathrm{COM}}^{A \rightarrow B}$ and $\pi_{\mathrm{COM}}^{B \rightarrow A}$ does not $\left(a^{\prime}, s^{\prime}\right)$-BQS-UC-emulate the composition of $\mathcal{F}_{\mathrm{COM}}^{A \rightarrow B}$ and $\mathcal{F}_{\mathrm{COM}}^{B \rightarrow A}$ (for any parameters $a^{\prime}, s^{\prime}$ ). To convert this into an example of a protocol that does not even compose with itself, just consider the protocol $\pi_{\mathrm{COM}}^{A \leftrightarrow B}$ in which Bob may choose whether $\mathcal{F}_{\mathrm{COM}}^{A \rightarrow B}$ or $\mathcal{F}_{\mathrm{COM}}^{B \rightarrow}$ should be executed. It might be possible to make $\pi_{\mathrm{COM}}^{A \leftrightarrow B}$ self-composable by adding suitable tags inside the messages, but the definition of BQS-UC-security does not enforce this.

Although BQS-UC-security does not guarantee for concurrent selfcomposability, individual protocols may have this property. In order to formulate this, we introduce the concept of the multi-session variant of a protocol. Given a protocol $\pi$ and a polynomially-bounded $n$, we define $\pi^{n}$ to be the protocol that executes $n$ instances of $\pi$ concurrently.

\footnotetext{
${ }^{7}$ In the proof, the difficulty arises from a hybrid argument where the protocol $\pi$ is executed together in one network with the protocol $\rho$ and the corresponding simulator. Since the simulator may use more quantum memory than $\pi$ is resistant against, we cannot guarantee security of $\pi$ in this hybrid setting and the proof cannot proceed.
} 
Then, from Theorem 2, we immediately get the following corollary:

Corollary 3. Let $\pi$ and $\sigma$ be quantum-polynomial-time protocols and $\mathcal{F}$ and $\mathcal{G}$ be quantum-polynomial-time functionalities. Let $n, m \geq 0$ be integers (depending on the security parameter). Assume that $\sigma$ invokes at most $m$ subprotocol instances. Assume that $\pi^{n m}(a, s)$-BQS-UC-emulates $\mathcal{F}^{n m}$ and that $\left(\sigma^{\mathcal{F}}\right)^{n}$ $\left(a-n \mathrm{QM}(\sigma)+s, s^{\prime}\right)$-BQS-UC-emulates $\mathcal{G}^{n}$. Then $\left(\sigma^{\pi}\right)^{n}\left(a-n \mathrm{QM}(\sigma), s+s^{\prime}\right)$ $B Q S$-UC-emulates $\mathcal{G}^{n}$.

\section{Commitments}

\subsection{Extractable Commitments}

In this section, we present the notion of online-extractable commitments in the BQS model. These will be used as a building block for constructing BQS-UC commitments in the next section.

Definition 4 (( $\varepsilon, a)$-BQS-hiding). Given a commitment protocol $\pi$ with sender Alice and recipient Bob, and an adversary $B^{\prime}$ corrupting Bob, we denote with $\left\langle A(m), B^{\prime}\right\rangle_{B^{\prime}}$ the output of $B^{\prime}$ in an interaction between Alice and $B^{\prime}$ where Alice commits to $m$.

We call $\pi(\varepsilon, a)$-BQS-hiding iff for all a-memory bounded $B^{\prime}$ and all $m_{1}, m_{2} \in$ $M$, we have that $\left|\operatorname{Pr}\left[\left\langle A\left(m_{1}\right), B^{\prime}\right\rangle_{B^{\prime}}=1\right]-\operatorname{Pr}\left[\left\langle A\left(m_{2}\right), B^{\prime}\right\rangle_{B^{\prime}}=1\right]\right| \leq \varepsilon$. Here $M$ is the message space of the commitment scheme.

Instead of the binding property, we will need a stronger property: onlineextractability. This property guarantees that there is a machine (the extractor) that, when running as the recipient of the commit protocol, is able to output the committed value $V$ already after the commit phase. This extractor should be indistinguishable from an honest recipient. Note that this does not contradict $(\varepsilon, a)$-BQS-hiding since we allow the extractor's quantum memory to contain more than $a$ qubits. For our purposes, we will only need a definition of onlineextractability that does not impose a memory bound on the adversary. We do, however, make the memory bound $s$ of the extractor explicit.

Definition 5 ( $(\varepsilon, s)$-online-extractable). Given a commitment protocol $\pi$ with sender Alice and recipient Bob, an extractor is a machine $B_{S}$ that, after the commit phase, gives an output $V^{\prime}$ and then executes the (honest) code of Bob for the open phase and outputs a value $V$ (the accepted value). (In particular, $B_{S}$ needs to provide an initial state for the program of the open phase of Bob that matches the interaction so far.) We write $V=\perp$ if the open phase fails.

For an adversary $A^{\prime}$, we denote with $\left\langle A^{\prime}, B\right\rangle_{A^{\prime}}\left(\left\langle A^{\prime}, B_{S}\right\rangle_{A^{\prime}}\right)$ the output of $A^{\prime}$ in an interaction between $A^{\prime}$ and $B o b\left(B_{S}\right)$ where $A^{\prime}$ is given $V$ after Bob $\left(B_{S}\right)$ terminates.

We call $\pi(\varepsilon, s)$-online-extractable iff there exists an s-memory bounded quantum-polynomial-time extractor $B_{S}$ such that for all adversaries $A^{\prime}$, we have that $\left|\operatorname{Pr}\left[\left\langle A^{\prime}, B\right\rangle_{A^{\prime}}=1\right]-\operatorname{Pr}\left[\left\langle A^{\prime}, B_{S}\right\rangle_{A^{\prime}}=1\right]\right| \leq \varepsilon$ and in an interaction of $A^{\prime}$ and $B_{S}$, we have $\operatorname{Pr}\left[V \notin\left\{V^{\prime}, \perp\right\}\right] \leq \varepsilon$. 
Theorem 6 (Online-extractable commitments). For any polynomiallybounded integers $a$ and $\ell$, there is a constant-round 0-memory bounded $(\varepsilon, a)$-BQS-hiding $(\varepsilon, s)$-online-extractable commitment scheme $\pi$ for some exponentially-small $\varepsilon$ and some polynomially-bounded $s$. The message space of $\pi$ is $M=\{0,1\}^{\ell}$.

A protocol with the properties from Theorem 6 was constructed in [12. They did not, however, show that it is online-extractable. In the full version [16] we show how their proof of the binding property can be extended to online-extractability.

\subsection{BQS-UC Commitments}

In this section, we present a commitment scheme $\pi_{\mathrm{COM}}$ that is BQS-UC-secure for memory bound $a$ and for $n$ concurrent instances of $\pi_{\mathrm{COM}}$. The parameters $a$ and $n$ can be arbitrary, but $\pi_{\mathrm{COM}}$ depends on them. To state our result, we first define the ideal functionality for commitments.

Definition 7 (Commitment). Let $A$ and $B$ be two parties. The functionality $\mathcal{F}_{\mathrm{COM}}^{A \rightarrow B, \ell}$ behaves as follows: Upon (the first) input (commit, $x$ ) with $x \in\{0,1\}^{\ell(k)}$ from $A$, store $x$ and send committed to $B$. Upon (the first) input open from $A$ send (open, $x$ ) to $B$ (unless $x$ is still undefined). All communication/input/output is classical. We call $A$ the sender and $B$ the recipient.

Note that this definition also defined the behavior of the functionality in the case where $A$ or $B$ is corrupted. In this case, the adversary (or simulator) is allowed to send/receive the inputs/outputs in the name of $A$ or $B$, respectively. For example, if $A$ is corrupted, the adversary can decide when to commit to what message and when to open.

Intuition. The protocol $\pi_{\mathrm{COM}}$ is depicted in Figure 1. Before we prove its security, we first explain the underlying intuition. In order to prove the BQS-UCsecurity of $\pi_{\mathrm{COM}}$, it is necessary to construct a simulator (that may use more quantum memory than the adversary) that achieves the following: When being in the role of the recipient, the simulator is able to extract the commitment after the commit phase. When being in the role of the sender, the simulator should be able to open the commitment to any value of his choosing (equivocality). The first requirement can easily be achieved by using the online-extractable commitment scheme from Theorem 6. That scheme, however, is not equivocal. In order to make our protocol equivocal, we intentionally weaken the binding property of the commitment. Instead of committing to a single value $v$, the sender commits using a commitment scheme $C_{2}$ to random values $\underline{R}:=R_{1}, \ldots, R_{m}$. Then he sends $v \oplus F(\underline{R})$ with $F$ being a universal hash function and sends the syndrome $\sigma$ of $\underline{R}$ with respect to a suitable linear code. In the open phase, the sender does not open all commitments $R_{i}$, but instead just sends $\underline{R}$ to the recipient. The recipient chooses a test set $T$, and the sender opens $R_{i}$ for $i \in T$. The modified scheme is still binding: Assume the sender wishes to be able to open the commitment with two different values. Then he has to find values $\underline{R}^{\prime} \neq \underline{R}$ that both pass the recipients checks in the open phase. If $\underline{R}^{\prime}$ differs from $\underline{R}$ in many 
Parameters: Integers $\ell$ (the length of the committed value), $m, c<m, b, d, \kappa<m$. A $b$-block $(m, \kappa, d)$-linear cod\& 8 where $\mathbf{S}(\omega) \in\{0,1\}^{(m-\kappa) b}$ denotes the syndrome of a codeword $\omega \in\{0,1\}^{m b}$. A family $\mathbf{F}$ of strongly universal hash functions $F:\{0,1\}^{m b} \rightarrow$ $\{0,1\}^{\ell}$. All parameters may depend on the security parameter $k$.

Subprotocols: A commitment scheme $C_{1}$ with sender Bob, and a commitment scheme $C_{2}$ with sender Alice, both 0-memory bounded (not using quantum memory)

Parties: The sender Alice $A$ and the recipient Bob $B$.

Inputs: In the commit phase, Alice gets (commit, $v$ ) with $v \in\{0,1\}^{\ell}$. In the open phase, Alice gets open. Bob gets no inputs.

\section{Commit phase:}

C1. Bob picks a random $T \subseteq\{1, \ldots, m\}$ with $\# T=c$. Then Bob commits to $T$ using $C_{1}$. (We assume some encoding of sets $T$ that does not allow to encode sets with $\# T \neq c$.)

C2. Alice picks $R_{1}, \ldots, R_{m} \in\{0,1\}^{b}$. For each $i$, Alice commits to $R_{i}$ using $C_{2}$. (The commitments may be performed concurrently.)

C3. Alice picks a hash function $F \leftarrow \mathbf{F}$, computes $p:=v \oplus F\left(R_{1}\|\ldots\| R_{m}\right)$, computes the syndrome $\sigma:=\mathbf{S}\left(R_{1}\|\ldots\| R_{m}\right)$, and sends $(F, \sigma, p)$ to Bob. (This may be done concurrently with the commitments to $R_{i}$.)

C4. Bob outputs committed.

\section{Open phase:}

O1. Alice sends $R_{1}\|\ldots\| R_{m}$ to Bob.

O2. Bob opens $T$ using $C_{1}$.

O3. For each $i \in T$, Alice opens $R_{i}$ using $C_{2}$. (The open phases may be executed concurrently.)

O4. Bob checks that the values $R_{i}$ sent by Alice match the values $R_{i}$ opened by Alice for all $i \in T$, and that $\sigma=\mathbf{S}\left(R_{1}\|\ldots\| R_{m}\right)$.

O5. Bob computes $v:=p \oplus F\left(R_{1}\|\ldots\| R_{m}\right)$ and outputs (open, $v$ ). (I.e., Bob accepts the opened value $v$.)

Fig. 1. Our commitment protocol $\pi_{\mathrm{COM}}$

blocks $R_{i}$, with high probability the verifier will require that one of these $R_{i}$ is opened and the sender will be caught. If $\underline{R}^{\prime}$ and $\underline{R}$ differs in only few blocks, then $\underline{R}-\underline{R}^{\prime}$ has a low Hamming weight and is not in the code. Hence the syndrome of $\underline{R}-\underline{R}^{\prime}$ is not zero, and, since the code is linear, the syndromes of $\underline{R}^{\prime}$ and $\underline{R}$ cannot both equal $\sigma$. Thus the sender is caught, too. Furthermore, our scheme is online-extractable if $C_{2}$ is online-extractable since the simulator can extract the committed values $\underline{R}$. However, we have not yet achieved the equivocality. In order to open the commitment to a different value, the sender needs to know $T$ before sending $\underline{R}^{\prime}$. To achieve this, the recipient commits to $T$ before the commit phase (using an online-extractable commitment scheme $C_{2}$ ). A simulator wishing to change the value of the commitment simply extracts $T$. Then he knows which $R_{i}$ can be changed without being detected and can thus change $F\left(R_{1}, \ldots, R_{m}\right)$ to any value he wishes.

${ }^{8}$ That is, a code where the code words consist of $m$ blocks of $b$ bit, that contains $2^{b \kappa}$ codewords, and where every non-zero codeword contains at least $d$ nonzero blocks.

${ }^{9}$ Note that this does not refer to the memory bound of the adversary. We only state that honest Alice and Bob do not need to use quantum memory in $C_{1}$ and $C_{2}$. 
Difficulties with Concurrent Composition. The main difficulty in showing the BQC-UC-security of $\pi_{\mathrm{COM}}$ lies in coping with the fact that several (say $n$ ) instances of $\pi_{\mathrm{COM}}$ might run concurrently. Consider for example the case that Alice is corrupted. In this case, the adversary may produce the $C_{2}$-commitments to $R_{1}, \ldots, R_{m}$ in $n$ instances of Alice. The simulator needs to run the $n m$ extractors to extract these commitments. Each of these extractors needs some quantum memory $s_{2}$. Thus our simulator needs $n m s_{2}$ bits of quantum memory. On the other hand, we need to make sure that the $C_{1}$-commitments to $T$, produced by the simulator, are hiding. $C_{1}$ needs to be hiding against $a_{1}$-bounded adversaries with $a_{1}>n m s_{2} \geq s_{2}$ (because we cannot be sure that the memory used by the simulator is not misused by the adversary). But then the extractor for $C_{1}$ needs to use $s_{1}>a_{1}$ qubits; otherwise the adversary could run the extractor to break the protocol. Similarly, we can see that when Bob is corrupted, $C_{2}$ needs to be hiding against $a_{2}$-memory bounded adversaries with $a_{2}>n s_{1} \geq s_{1}$, and $s_{2}>a_{2}$. Thus we need $a_{1}>s_{2}>a_{2}>s_{1}>a_{1}$ which is impossible.

Solving the Difficulties. The way out is to carefully track the memory used by the simulators; it turns out that in the proof of security against corrupted Alice, we can make sure that the adversary is not able to "misuse" the memory of the simulator. When Alice is corrupted, we need to construct a simulator that extracts the values $v$ of $n$ concurrent commitments produced by Alice, while being indistinguishable from an execution of the honest recipient Bob. More precisely, we show that the simulator is indistinguishable if $C_{1}$ is $a_{1}$-BQS-hiding and $C_{2}$ is $s_{2}$-online-extractable and environment and adversary are $a_{1}$-memorybounded. We do not require that $a_{1}>s_{2}$, thus breaking the above-mentioned circularity in the choices of $a_{1}, s_{1}, a_{2}, s_{2}$.

Let $B^{*}$ be defined like the honest Bob, except that instead of honestly running the recipient's code for $C_{2}, B^{*}$ runs the extractor $B_{S}$ for $C_{2}$ to extract the committed values $\underline{R}$ in the $C_{2}$-commitments. From $\underline{R}, B^{*}$ computes a guess $v^{\prime}$ for the committed value $v$. $B^{*}$ does not, however, use this guess at any point.

First, note that $B^{*}$ is indistinguishable from honest Bob: This follows from the fact that the extractor for $C_{2}$ is indistinguishable from the recipient for $C_{2}$. Furthermore, as discussed in the section "Intuition" above, extracting $v$ will be successful as long as Alice does not learn anything about $T$, i.e., as long as $C_{1}$ is hiding. But $C_{1}$ is only $a_{1}$-BQS-hiding. And $B^{*}$ uses $m s_{2}$ qubits to run the extractors for $C_{2}$, so the total memory used in the network is $a_{1}+m s_{2}$ which is beyond the memory bound tolerated by $C_{1}$. Fortunately, however, honest Bob runs the recipient of $C_{2}$ after the end of the commit phase and before the beginning of the open phase of $C_{1}$. Thus, from the point of view of $C_{1}$, the extractors are executed within a single atomic computation. And we defined BQS-hiding to hold even if the adversary uses unlimited memory within a single activation. Thus the $m s_{2}$ qubits used by the extractors do not break the hiding property, and we get that $B^{*}$ guesses the right $v^{\prime}$ with overwhelming probability.

This argument does, however, only work when a single instance of $B^{*}$ is executed. If several instances of $B^{*}$ are executed, one instance may run the commit or open phase of $C_{1}$ concurrently with another instance's extractors. 
Two show that $n$ concurrent instances of $B^{*}$ extract successfully, we use the following argument: For each $j$, we have that if only the $j$-th Bob instance is replaced by $B^{*}$, then $B^{*}$ extracts correctly. Furthermore, Bob and $B^{*}$ are indistinguishable, thus if we replace all the other instances of Bob by $B^{*}$, the $j$-th instance still extracts correctly. Thus, for any $j$, if there are $n$ instances of $B^{*}$, then the $j$-th instance extracts correctly. Thus all instances of $B^{*}$ extract correctly. And the instances of $B^{*}$ are indistinguishable from the instances of Bob.

Finally, we can construct a simulator that runs $B^{*}$ instead of Bob and uses the value $v^{\prime}$ extracted by $B^{*}$ as input to the commitment functionality. Since $v=v^{\prime}$ with overwhelming probability, this simulator is successful.

Thus we have shown that $a_{1}$ can be chosen independently of $s_{2}$. This allows to break the circularity in the choices of $a_{1}, s_{1}, a_{2}, s_{2}$ : We first start with an arbitrary $a=a_{1}$. Then we pick an arbitrary $a_{1}$-BQS-hiding and $s_{1}$-online-extractable $C_{1}$, and then an arbitrary $a_{2}:=a+n s_{1}$-BQS-hiding and $s_{2}$-online-extractable commitment $C_{2}$. For the case of corrupted Bob, we then construct a simulator that uses $n s_{1}$ qubits and is secure against $a=a_{2}-n s_{1}$-memory bounded environments and adversaries. And for the case of corrupted Alice, using the argument above, we get a simulator that uses $n m s_{2}$ qubits and is secure against $a=a_{1}$-bounded environments and adversaries.

The Analysis. We proceed with the formal analysis of $\pi_{\mathrm{COM}}$. We first consider the case where the recipient is corrupted.

Lemma 8. Assume that $\varepsilon, \delta$ are negligible, $n, c$ are polynomially-bounded, and $2 \kappa b-m b-2 c b-\ell$ is superlogarithmic (in the security parameter $k$ ). Assume that $C_{1}$ is $\left(\varepsilon, s_{1}\right)$-online-extractable and $C_{2}$ is $\left(\delta, a+n s_{1}\right)$-BQS-hiding. Assume that $\mathbf{F}$ is a family of affine strongly universal hash functions.

Then $\pi_{\mathrm{COM}}^{n}\left(a, n s_{1}\right)$-BQS-UC-emulates $\left(\mathcal{F}_{\mathrm{COM}}^{A \rightarrow B, \ell}\right)^{n}$ for corrupted recipient $B$.

Proof. First, we describe the structure of the real and ideal model in the case that the party $B$ (Bob) is corrupted:

In the real model, we have the environment $\mathcal{Z}$, the adversary $\mathrm{Adv}$, the honest party $A$ (Alice), the corruption party $B^{C}$. The adversary controls the corruption party $B^{C}$, so effectively he controls the communication between Alice and Bob. The environment provides Alice's inputs (commit, $v$ ) and open. See Figure 2(a).

In the ideal model, we have the environment $\mathcal{Z}$, the simulator Sim (to be defined below), the dummy-party $\tilde{A}$, the corruption party $B^{C}$, and the commitment functionality $\mathcal{F}_{\mathrm{COM}}$. The inputs (commit, $v$ ) and open of $\mathcal{F}_{\mathrm{COM}}$ are provided by the dummy-party $\tilde{B}$ and thus effectively by the environment $\mathcal{Z}$. The simulator Sim controls the corruption party $B^{C}$ and hence gets the outputs committed and (open, $v$ ) of $\mathcal{F}_{\mathrm{COM}}$. See Figure 2 (b).

Fix an adversary Adv. To show Lemma 8, we need to find a simulator Sim with $\mathrm{QM}(\operatorname{Sim}) \leq n s_{1}$ such that, for any environment $\mathcal{Z}$ with $\mathrm{QM}(\mathcal{Z})+\mathrm{QM}(\mathrm{Adv}) \leq$ $a$, the real model and the ideal model are negligible-close. This simulator is described in Figure 3. We use the abbreviations $\underline{R}:=R_{1}\|\ldots\| R_{m}$ and $\underline{R}^{\prime}:=$ $R_{1}^{\prime}\|\ldots\| R_{m}^{\prime}$. 
(a)

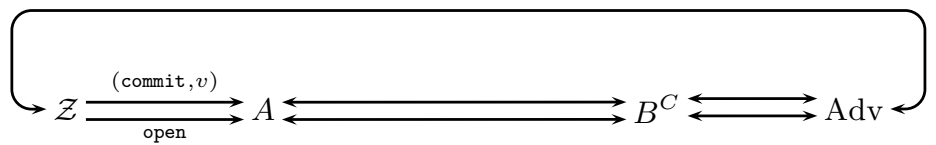

(b)

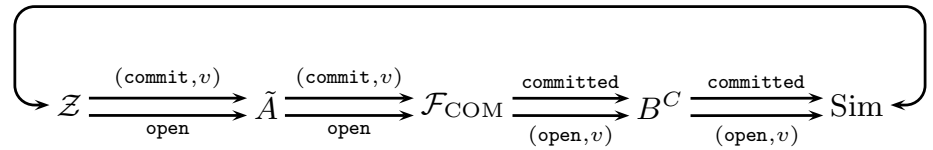

Fig. 2. Networks occurring in the proof of Lemma 8

To show that the real and the ideal model are negligible-close, we start with the real model, and change the machines in the real model step-by-step until we end up with the ideal model. In each step, we show that the network before and after that step are negligible-close.

Game 1. We change the machine $A$ as follows: Instead of executing the program of the honest recipient of $C_{1}, A$ executes the extractor $A_{S}$.

Let $T^{\prime}$ denote the extracted value. The modified $A$ does not use $T^{\prime}$. Since there are up to $n$ copies of $A$, and since $C_{1}$ is $\left(\varepsilon, s_{1}\right)$-online-extractable, the real model and Game 1 are $n \varepsilon$-close.

Game 2. We change the machine $A$ to abort if the opening of $T$ succeeds and reveals a value $T \neq T^{\prime}$.

Since $C_{1}$ is $\left(\varepsilon, s_{1}\right)$-online-extractable, in each instance of $A$, this happens with probability at most $\varepsilon$, thus Game 1 and Game 2 are $n \varepsilon$-close.

Notice that the only machines that use quantum memory in Game 2 are $\mathcal{Z}, \mathrm{Adv}$, and $n$ copies of $A_{S}$. Since $A_{S}$ is $s_{1}$-memory bounded, and $\mathrm{QM}(\mathcal{Z})+$ $\mathrm{QM}(\mathrm{Adv}) \leq a$ we have that the total amount of quantum memory used in Game 2 is bounded by $a+n s_{1}$.

Game 3. We change the machine $A$ to commit to $0^{b}$ instead of $R_{i}$ for each $i \notin T^{\prime}$.

To see that Game 2 and Game 3 are negligible-close, we introduce an intermediate hybrid game, Game 3 ; , in which only the first $j$ of the commitments to $R_{i}, i \notin T$ are replaced by commitments to $0^{b}$. Since at most $a+n s_{1}$ qubits of quantum memory are used in Game 2 and therefore also in Game 3j; , and since the $C_{2}$-commitments to $R_{i}, i \notin T$ are never opened, from the fact that $C_{2}$ is $\left(\delta, a+n s_{1}\right)$-BQS-hiding it follows that Game 3; and Game $3 j+1$ are $\delta$ close. Note that there are, in the whole game, up to $n$ copies of $A$ and thus up to $n c C_{2}$-commitments to some $R_{i}, i \notin T$. Thus Game 2 Game 3, and Game 3 $=$ Game 3 $n_{c}$ are $n c \delta$-close. 


\section{Commit phase (on input committed):}

- When Bob commits to $T$ using $C_{1}$, the simulator runs the extractor $A_{S}$ for $C_{1}$ instead of the honest recipient's program. (Since $C_{1}$ has recipient Alice, we write $A_{S}$, not $B_{S}$.) Let $T^{\prime}$ denote the value extracted by $A_{S}$.

- The simulator picks $R_{1}, \ldots, R_{m} \in\{0,1\}^{b}$. For each $i$, Sim commits (honestly) to $R_{i}$ (if $i \in T$ ) or to $0^{b}$ (if $i \notin T$ ) using $C_{2}$.

- Sim picks a hash function $F \stackrel{R}{\leftarrow} \mathbf{F}$, picks a random $p \stackrel{R}{\leftarrow}\{0,1\}^{\ell}$, computes the syndrome $\sigma:=\mathbf{S}(\underline{R})$, and sends $(F, \sigma, p)$ to Bob.

Open phase (on input (open, $v$ ) with $v \in\{0,1\}^{\ell}$ ):

- Sim picks $\underline{R}^{\prime} \in\left\{\underline{R}^{\prime}: \forall i \in T^{\prime} \cdot R_{i}=R_{i}^{\prime}, \sigma=\mathbf{S}\left(\underline{R}^{\prime}\right), p \oplus F\left(\underline{R}^{\prime}\right)=v\right\}$ uniformly 10 .

- Sim sends $\underline{R}^{\prime}$ to Bob.

- Sim waits for Bob to open $T$ using $C_{1}$. If $T \neq T^{\prime}$, Sim aborts.

- For each $i \in T^{\prime}$, Sim (honestly) opens $R_{i}$ using $C_{2}$.

Fig. 3. Simulator Sim for the case of corrupted Bob. The program described in this figure is executed for each instance of the $n$ instances of $\pi_{\mathrm{COM}}$. Communication with Bob is sent to an internally simulated instance of the adversary Adv.

Game 4. We modify $A$ to set $\underline{R}^{\prime}:=\underline{R}$ and to send $\underline{R}^{\prime}$ instead of $\underline{R}$ to Bob in step O[1]

This modification is for notational purposes only, Game 3 and Game 4 are perfectly close.

Game 5. We modify the way $A$ chooses $F, \underline{R}, \underline{R}^{\prime}, \sigma, p$ : In Game 4 we have $F \stackrel{R}{\leftarrow}$ $\mathbf{F}, \underline{R} \stackrel{R}{\leftarrow}\{0,1\}^{m b}, \sigma:=\mathbf{S}(\underline{R}), p:=v \oplus F(\underline{R}), \underline{R}^{\prime}:=\underline{R}$. (We call this distribution $\mathcal{D}_{1}$.) In Game 5 we use $F \stackrel{R}{\leftarrow} \mathbf{F}, \underline{R} \stackrel{R}{\leftarrow}\{0,1\}^{m b}, p \stackrel{R}{\leftarrow}\{0,1\}^{\ell}, \sigma:=\mathbf{S}(\underline{R}), \underline{R}^{\prime} \stackrel{R}{\leftarrow}$ $\left\{\underline{R}^{\prime}: \forall i \in T^{\prime} \cdot R_{i}=R_{i}^{\prime}, \sigma=\mathbf{S}\left(\underline{R}^{\prime}\right), p \oplus F\left(\underline{R}^{\prime}\right)=v\right\}=: \mathcal{R}_{F, \underline{R}, p}$. (We call this distribution $\mathcal{D}_{2}$.)

To show that Game 4 and Game 5 are negligible-close, we use the following claim:

Claim 1. Let $R_{T}:=\left(R_{i}\right)_{i \in T}$. For any $v \in\{0,1\}^{\ell}$, the statistical distance between $\left(F, R_{T}, \underline{R}^{\prime}, \sigma, p\right)$ chosen according to $\mathcal{D}_{1}$ and $\left(F, R_{T}, \underline{R}^{\prime}, \sigma, p\right)$ chosen according to $\mathcal{D}_{2}$ is at most $2^{c b+m b / 2+\ell / 2-\kappa b-1}$.

The proof of this claim uses the fact that $p$ is the result of applying $F$ to a random variable $\underline{R}$ with high min-entropy. Due to the leftover-hash-lemma [9], $p$ is indistinguishable from randomness. We refer to the full version [16] for the proof. Using Claim 1 and the fact that we have $n$ instances of $A$, we immediately get that Game 4 and Game 5 are $n\left(2^{c b+m b / 2+\ell / 2-\kappa b-1}\right)$-close because the values $\left(R_{i}\right)_{i \notin T}$ are never used by $A$ (except indirectly through $\underline{R}^{\prime}, \sigma$, and $p$ ).

Finally, note that by construction of Sim, Game 5 and the ideal model are perfectly close. Thus the real and the ideal model are $\gamma$-close with $\gamma:=2 n \varepsilon+$

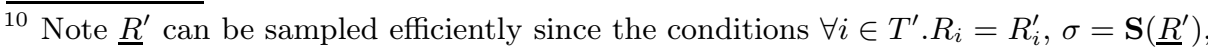
and $p \oplus F\left(\underline{R}^{\prime}\right)=v$ are a system of linear equations. This uses that $\mathbf{S}$ is the syndrome of a linear code, and that $\mathbf{F}$ is a family of affine functions. 
(a)

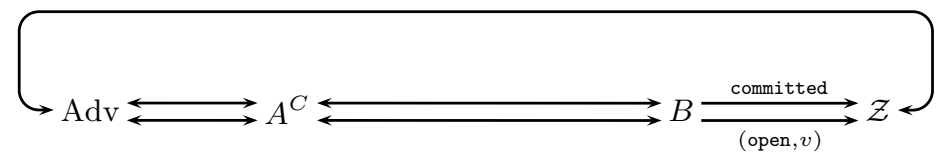

(b)

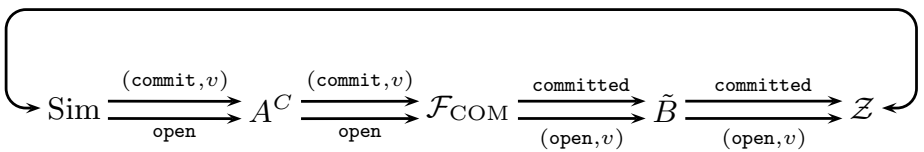

Fig. 4. Networks occurring in the proof of Lemma 9

$n c \delta+n\left(2^{c b+m b / 2+\ell / 2-\kappa b-1}\right)$. Since $\varepsilon, \delta$ are negligible, and $n, c$ are polynomiallybounded, and $2 \kappa b-m b-2 c b-\ell$ is superlogarithmic, we have that $\gamma$ is negligible. Thus $\pi_{\mathrm{COM}}^{n}\left(a, n s_{1}\right)$-BQS-UC-emulates $\left(\mathcal{F}_{\mathrm{COM}}^{A \rightarrow B, \ell}\right)^{n}$ in the case of corrupted Bob.

Lemma 9. Assume that $\varepsilon, \delta$ are negligible, $n$ is polynomially-bounded, and $\left(1-\frac{d}{m}\right)^{c}$ is negligible (in the security parameter $k$ ). Assume that $C_{1}$ is $(\varepsilon, a)$ $B Q S$-hiding and that $C_{2}$ is $\left(\delta, s_{2}\right)$-online-extractable. Assume that the code with syndrome $\mathbf{S}$ has efficient error-correction.

Then $\pi_{\mathrm{COM}}^{n}\left(a, n_{m} s_{2}\right)$-BQS-UC-emulates $\left(\mathcal{F}_{\mathrm{COM}}^{A \rightarrow B, \ell}\right)^{n}$ for corrupted sender $A$.

Proof. First, we describe the structure of the real and the ideal model in the case that the party $A$ (Alice) is corrupted:

In the real model, we have the environment $\mathcal{Z}$, the adversary Adv, the corruption party $A^{C}$, and the honest party $B$ (Bob). The adversary controls the corruption party $A^{C}$, so effectively he controls the communication between Alice and Bob. The environment gets Bob's outputs committed and (open, $v$ ). See Figure 4 (a).

In the ideal model, we have the environment $\mathcal{Z}$, the simulator Sim (to be defined below), the corruption party $A^{C}$, the dummy-party $\tilde{B}$, and the commitment functionality $\mathcal{F}_{\mathrm{COM}}$. The inputs (commit, $v$ ) and open of $\mathcal{F}_{\mathrm{COM}}$ are provided by the corruption party $A^{C}$ and thus effectively by the simulator Sim. The environment $\mathcal{Z}$ controls the dummy-party $\tilde{B}$ and hence gets the outputs committed and (open, $v$ ) of $\mathcal{F}_{\mathrm{COM}}$. See Figure 4 (b).

Fix an adversary Adv. To show Lemma 9, we need to find a quantumpolynomial-time simulator Sim with $\mathrm{QM}(\mathrm{Sim}) \leq n m s_{2}$ such that, for any environment $\mathcal{Z}$ with $\mathrm{QM}(\mathcal{Z})+\mathrm{QM}(\mathrm{Adv}) \leq a$, the real model and the ideal model are negligible-close. This simulator is described in Figure 5. Note that Sim is quantum-polynomial-time: The extractor $B_{S}$ is quantum-polynomial-time by definition, and computing $\underline{R}^{*}$ is possible in polynomial-time because the code with syndrome $\mathbf{S}$ has efficient error-correction. Since $C_{2}$ is $\left(\delta, s_{2}\right)$-onlineextractable and Sim uses $m$ instances of $B_{S}$ per copy of $B, \mathrm{QM}(\operatorname{Sim}) \leq n m s_{2}$. We use the abbreviations $\underline{R}:=R_{1} \| \ldots R_{m}$ and similarly for $\underline{R}^{\prime}$ and $\underline{R}^{*}$. 


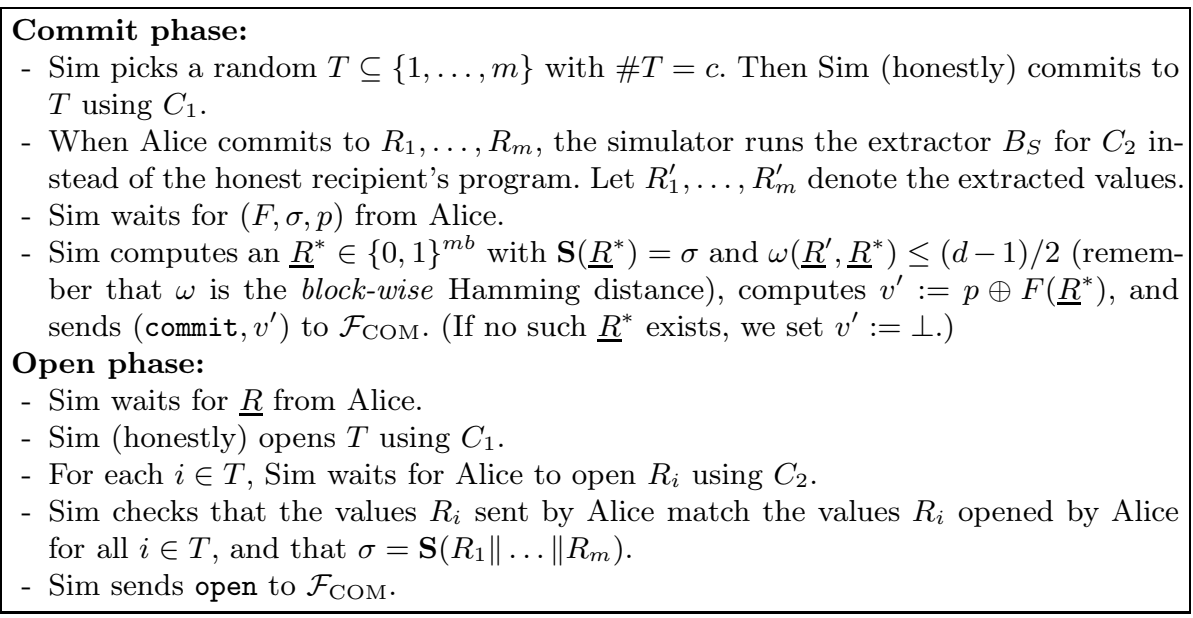

Fig. 5. Simulator Sim for the case of corrupted Alice. The program described in this figure is executed for each instance of the $n$ instances of $\pi_{\mathrm{COM}}$. Communication with Alice is sent to an internally simulated instance of the adversary Adv.

Before we proceed, we introduce two variants of the honest recipient $B$. The machine $B^{*}$ behaves like $B$, but when Alice commits to $R_{1}, \ldots, R_{m}$ using $C_{2}$, $B^{*}$ runs the extractor $B_{S}$ for $C_{2}$ instead of the honest recipient's program. Call the extracted values $R_{1}^{\prime}, \ldots, R_{m}^{\prime}$. Further, $B^{*}$ computes an $\underline{R}^{*}$ with $\mathbf{S}\left(\underline{R}^{*}\right)=\sigma$ and $\omega\left(\underline{R}^{\prime}, \underline{R}^{*}\right) \leq(d-1) / 2$ and then computes $v^{\prime}:=p \oplus F\left(\underline{R}^{*}\right)$. (If no such $\underline{R}^{*}$ exists, $v^{\prime}:=\perp$.) In the open phase, $B^{*}$ behaves like $B$. In particular, $B^{*}$ outputs (open, $v$ ), not (open, $v^{\prime}$ ). That is, $v^{\prime}$ is computed but never used.

The machine $B^{+}$behaves like $B^{*}$, but outputs (open, $v^{\prime}$ ) instead of (open, $v$ ).

By definition of online-extractability, and since $B^{*}$ does not use the value extracted by $B_{S}$, we have that $B$ and $B^{*}$ are $\delta$-indistinguishable. More precisely, for any network $S$, we have that $S \cup\{B\}$ and $S \cup\left\{B^{*}\right\}$ are $\delta$-close. Since onlineextractability was defined with respect to non-memory bounded adversaries, this holds even if $S$ is not memory bounded.

As in Lemma 8, we proceed by investigating a sequence of games.

Game 1. In the game Game 1 , the $j$-th instance of $B$ is replaced by an instance of $B^{*}$. (Note: only one instance is replaced, not the first $j$ instances.)

We use the following claim:

Claim 2. Let $S$ be an a-memory bounded network. In an execution of $S \cup\left\{B^{*}\right\}$, let $v, v^{\prime}$ denote the values $v, v^{\prime}$ computed by $B^{*}$. Then $\operatorname{Pr}\left[v \notin\left\{v^{\prime}, \perp\right\}\right] \leq \varepsilon+(1-$ $\left.\frac{d}{m}\right)^{c}:=\eta$.

We prove this claim below. Since $C_{1}$ and $C_{2}$ are 0 -memory bounded, we have that the machine $B$ is 0 -memory bounded. $\mathrm{QM}(\mathcal{Z})+\mathrm{QM}(\mathrm{Adv}) \leq a$. Thus we can apply Claim 2 to Game 1 . Hence in Game 1 $_{j}, \operatorname{Pr}\left[v_{j} \notin\left\{v_{j}^{\prime}, \perp\right\}\right] \leq \eta$ where $v_{j}, v_{j}^{\prime}$ are the values $v, v^{\prime}$ computed by $B^{*}$. We write $v_{j}:=\perp$ if the open phase fails or does not take place (and hence $v_{j}$ is not computed by $B^{*}$ ). 
Game 2. This game is defined like the real model, except that we use $n$ instances of $B^{*}$ instead of the $n$ instances of $B$.

Using the fact that $B$ and $B^{*}$ are $\delta$-indistinguishable, we get that the real model and Game 2 are $n \delta$-close.

Again using that $B$ and $B^{*}$ are $\delta$-indistinguishable, we get that $\mid \operatorname{Pr}\left[v_{j} \notin\right.$ $\left\{v_{j}^{\prime}, \perp\right\}:$ Game 1 $\left.] j\right]-\operatorname{Pr}\left[v_{j} \notin\left\{v_{j}^{\prime}, \perp\right\}\right.$ : Game 2] $\mid \leq(n-1) \delta$. Thus $\operatorname{Pr}\left[v_{j} \notin\right.$ $\left\{v_{j}^{\prime}, \perp\right\}:$ Game 2 $\leq \eta+(n-1) \delta$. Since this holds for all $j=1, \ldots, n$, we get:

$$
\operatorname{Pr}\left[\exists j . v_{j} \notin\left\{v_{j}^{\prime}, \perp\right\}: \text { Game 2] } \leq n \eta+n(n-1) \delta .\right.
$$

Game 3. This game is defined like the real model, except that we use $n$ instances of $B^{+}$instead of the $n$ instances of $B$.

Notice that Game 2 and Game 3 only differ in the fact that in Game 2 we use instances of $B^{*}$ and in Game 3 instances of $B^{+}$. By definition, $B^{*}$ and $B^{+}$only differ in the value they output: $B^{*}$ outputs (open, $v$ ) and $B^{+}$outputs (open, $v^{\prime}$ ). By (1), the probability that the values $v, v^{\prime}$ are different in some instance of $B^{*}$ is bounded by $n \eta+n(n-1) \delta$. Hence Game 2 and Game 3 are $(n \eta+n(n-1) \delta)$-close.

Finally, note that by construction of Sim, Game 3 and the ideal model are perfectly close. Thus the real and the ideal model are $\gamma$-close with $\gamma:=$ $n \delta+n \eta+n(n-1) \delta=n^{2} \delta+n \varepsilon+n\left(1-\frac{d}{m}\right)^{c}$. Since $\delta, \varepsilon$ are negligible, $n$ is polynomially-bounded, and $\left(1-\frac{d}{m}\right)^{c}$ is negligible, we have that $\gamma$ is negligible. Thus $\pi_{\mathrm{COM}}^{n}\left(a, n m s_{2}\right)$-BQS-UC-emulates $\left(\mathcal{F}_{\mathrm{COM}}^{A \rightarrow B, \ell}\right)^{n}$ in the case of corrupted Alice.

Proof of Claim 2, Let $\underline{R}, \underline{R}^{\prime}, \underline{R}^{*}$ and $T$ denote the corresponding values as computed by $B^{*}$. We abbreviate $R_{T}:=\left(R_{i}\right)_{i \in T}$ and $R_{T}^{\prime}:=\left(R_{i}^{\prime}\right)_{i \in T}$. By Bad we denote the event that $R_{T}=R_{T}^{\prime}$ and $\mathbf{S}(\underline{R})=\sigma$ and $\underline{R} \neq \underline{R}^{*}$. By construction of $B^{*}, v \neq \perp$ implies $R_{T}=R_{T}^{\prime}$ and $\mathbf{S}(\underline{R})=\sigma$. And $v \notin\left\{v^{\prime}, \perp\right\}$ implies $\underline{R} \neq \underline{R}^{*}$. Thus $v \notin\left\{v^{\prime}, \perp\right\}$ implies Bad. Therefore, to show Claim 2, it is sufficient to show $\operatorname{Pr}[B a d] \leq \eta$ in $S \cup\left\{B^{*}\right\}$. To show this, we again proceed using a sequence of games:

Game 4. An execution of $S \cup\left\{B^{*}\right\}$.

Game 5. We change $B^{*}$ to halt after receiving $\underline{R}$ from Alice.

Then $\operatorname{Pr}[$ Bad : Game 4] $=\operatorname{Pr}[$ Bad : Game 5].

Game 6. We change $B^{*}$ to commit to some (arbitrary) fixed value $T_{0}$ instead of committing to $T$.

We wish to apply the $(\varepsilon, a)$-BQS-hiding property of $C_{1}$ in order to show that $\mid \operatorname{Pr}\left[B a d:\right.$ Game 5] $-\operatorname{Pr}\left[B a d:\right.$ Game 6] $\mid \leq \varepsilon$. Let $B_{1}$ denote the sender in the commitment scheme $C_{1}$. By definition, to commit to $T$ (or $T_{0}$ ), $B^{*}$ internally runs $B_{1}$. We construct an adversary $A_{1}^{\prime}$ that interacts with $B_{1}$. This adversary simulates $S \cup\left\{B^{*}\right\}$ (with $B^{*}$ as in Game 5) except for the machine $B_{1}$ inside $B^{*}$. Note that in Game 5 only the commit phase of $C_{1}$ is executed. We let $A_{1}^{\prime}$ output 1 iff $B a d$ happens. We define $\hat{B}_{1}$ like $B_{1}$, except that $\hat{B}_{1}$ ignores its input and 
commits to $T_{0}$. Let $P$ be the probability that $A_{1}^{\prime}$ outputs 1 when running with $B_{1}$, and let $\hat{P}$ be the probability that $A_{1}^{\prime}$ outputs 1 when running with $\hat{B}_{1}$. By construction, $P=\operatorname{Pr}[B a d$ : Game 5] and $\hat{P}=\operatorname{Pr}[B a d$ : Game 6]. Thus we only have to show that $|P-\hat{P}| \leq \varepsilon$. To apply the $(\varepsilon, a)$-BQS-hiding property of $C_{1}$ we have to check that $A_{1}^{\prime}$ is $a$-memory bounded. $A_{1}^{\prime}$ simulates $\mathcal{Z}, \mathrm{Adv}$, and $B^{*}$. We have $\mathrm{QM}(\mathcal{Z})+\mathrm{QM}(\mathrm{Adv}) \leq a$ by assumption. But $B^{*}$ contains the extractor $B_{S}$ for $C_{2}$ which uses additional $s_{2}$ qubits of quantum memory. Yet, $B_{S}$ is executed after the end of the commit phase of $C_{1}$. That is, $B^{*}$ is executed within a single activation of $A_{1}^{\prime}$ (since $B_{1}$ is not activated any more after the commit phase). Note that, although $A_{1}^{\prime}$ might use more than $a$ qubits during the activation in which $B^{*}$ is simulated, it stores at most a qubits between activations. Thus $A_{1}^{\prime}$ is $a$-memory bounded (remember that our definition of " $a$-memory bounded" on page 470 only requires that the memory bound holds between activations). Hence $|P-\hat{P}| \leq \varepsilon$ and thus $\mid \operatorname{Pr}[\operatorname{Bad}:$ Game 5] $-\operatorname{Pr}[$ Bad : Game 6] $\mid \leq \varepsilon$.

Game 7 . We change $B^{*}$ to choose $T$ only after receiving $\underline{R}^{\prime}$.

Since $T$ is not used earlier by $B^{*}, \operatorname{Pr}[B a d:$ Game 6] $=\operatorname{Pr}[B a d$ : Game 7]. Fix values $\underline{R}, \underline{R}^{\prime}$ and $\sigma$ with $\mathbf{S}(\underline{R})=\sigma$. We distinguish two cases, depending on whether there exists an $\underline{R}^{*}$ with $\mathbf{S}\left(\underline{R}^{*}\right)=\sigma$ and $\omega\left(\underline{R}^{*}, \underline{R}^{\prime}\right) \leq(d-1) / 2$. Case " $\underline{R}^{*}$ exists": Since $\mathbf{S}$ is the syndrome of a b-block $(m, \kappa, d)$-linear code, $\mathbf{S}\left(\underline{R}-\underline{R}^{*}\right)=0$, hence $\underline{R}-\underline{R}^{*}$ is a codeword. Hence $\underline{R}=\underline{R}^{*}$ or $\omega\left(\underline{R}, \underline{R}^{*}\right) \geq d$. Using the triangle inequality and $\omega\left(\underline{R}^{*}, \underline{R}^{\prime}\right) \leq(d-1) / 2$, we get that $\underline{R}=\underline{R}^{*}$ or $\omega\left(\underline{R}, \underline{R^{\prime}}\right) \geq d-(d-1) / 2 \geq d / 2$. Case " $\underline{R}^{*}$ does not exist": Since no $\underline{R}^{*}$ with $\mathbf{S}\left(\underline{R}^{*}\right)=\sigma$ and $\omega\left(\underline{R}^{*}, \underline{R}^{\prime}\right) \leq(d-1) / 2$ exists, and since $\mathbf{S}(\underline{R})=\sigma$, we have that $\omega\left(\underline{R}, \underline{R}^{\prime}\right)>(d-1) / 2$. Hence $\omega\left(\underline{R}, \underline{R}^{\prime}\right) \geq d / 2$.

Thus, for any fixed choice of $\underline{R}, \underline{R}^{\prime}, \sigma$, we have $\mathbf{S}(\underline{R}) \neq \sigma$ or $\underline{R}=\underline{R}^{*}$ or $\omega\left(\underline{R}, \underline{R}^{\prime}\right) \geq d / 2$.

If $\underline{R}=\underline{R}^{*}$ or if $\mathbf{S}(\underline{R}) \neq \sigma$, the event $B a d$ does not occur by definition.

If $\omega\left(\underline{R}, \underline{R}^{\prime}\right) \geq d / 2$, we bound the probability of Bad occurring as follows: Let $D:=\left\{i: R_{i} \neq R_{i}^{\prime}\right\}$. Then, for random $T \subseteq[m]$ with $\# T=c$, we have $\operatorname{Pr}[B a d] \leq \operatorname{Pr}\left[R_{T}=R_{T}^{\prime}\right]=\operatorname{Pr}[T \cap D=\varnothing] \leq\left(1-\frac{\# D}{m}\right)^{c} \leq\left(1-\frac{d}{m}\right)^{c}$.

Thus for any fixed $\underline{R}, \underline{R}^{\prime}, \sigma$ we have $\operatorname{Pr}[$ Bad $] \leq\left(1-\frac{d}{m}\right)^{c}$. By averaging over the choice of $\underline{R}, \underline{R}^{\prime}, \sigma$, we get $\operatorname{Pr}[\operatorname{Bad}:$ Game 7$] \leq\left(1-\frac{d}{m}\right)^{c}$.

Summarizing, we have $\operatorname{Pr}\left[\operatorname{Bad}:\right.$ Game 4 $\leq \varepsilon+\left(1-\frac{d}{m}\right)^{c}=\eta$.

This shows Claim 2.

Using Reed-Solomon codes for $\mathbf{S}$, and the extractable commitments from Theorem 6 for $C_{1}$ and $C_{2}$, we can instantiate the parameters of $\pi_{\mathrm{COM}}$ to satisfy the conditions of Lemmas 8 and 9 . Thus we get the following theorem:

Theorem 10. Let $\ell, n$, and a be polynomially-bounded. Then there are choices for the parameters of $\pi_{\mathrm{COM}}$ and a polynomially-bounded integer $s$ such that $\pi_{\mathrm{COM}}$ is polynomial-time, constant-round and $\pi_{\mathrm{COM}}^{n}(a, s)-B Q S$-UC-emulates $\left(\mathcal{F}_{\mathrm{COM}}^{A \rightarrow B, \ell}\right)^{n}$. 
General Two-Party Computation. By combining known results [19 10 15, we get a constant-round protocol that quantum-UC-emulates any two-party functionality and uses only commitments from Alice to Bob. Combining this with our protocol $\pi_{\mathrm{COM}}$, we get our final result (for details see the full version [16]):

Theorem 11 (BQS two-party computation). Let $\mathcal{G}$ be a classical well-formed 11 probabilistic-polynomial-time functionality. Let $n$ and $a$ be polynomially-bounded. Then there is a polynomially-bounded $s$ and a constantround 0-memory bounded protocol $\pi_{\mathrm{bqs} 2 \mathrm{pc}}$ not invoking any functionality such that $\pi_{\text {bqs2pc }}^{n}(a, s)$-BQS-UC-emulates $\mathcal{G}^{n}$.

Acknowledgements. We thank Christian Schaffner and Jürg Wullschleger for valuable discussions. This work was funded by the Cluster of Excellence "Multimodal Computing and Interaction".

\section{References}

1. Ben-Or, M., Mayers, D.: General security definition and composability for quantum \& classical protocols (September 2004), http://xxx.lan.l.gov/abs/quant-ph/ 0409062

2. Bennett, C.H., Brassard, G.: Quantum cryptography: Public-key distribution and coin tossing. In: IEEE International Conference on Computers, Systems and Signal Processing 1984, pp. 175-179. IEEE Computer Society, Los Alamitos (1984)

3. Bennett, C.H., Brassard, G., Crépeau, C., Skubiszewska, M.H.: Practical quantum oblivious transfer. In: Feigenbaum, J. (ed.) CRYPTO 1991. LNCS, vol. 576, pp. 351-366. Springer, Heidelberg (1992)

4. Canetti, R.: Universally composable security: A new paradigm for cryptographic protocols. In: FOCS 2001, pp. 136-145. IEEE Computer Society, Los Alamitos (2001), full and revised version is [5]

5. Canetti, R.: Universally composable security: A new paradigm for cryptographic protocols. IACR ePrint Archive (January 2005), full and revised version of [4], http://eprint.tacr.org/2000/067.ps

6. Damgård, I., Fehr, S., Salvail, L., Schaffner, C.: Cryptography in the bounded quantum-storage model. In: FOCS 2005, pp. 449-458 (2005), a full version http: //arxiv.org/abs/quant-ph/0508222

7. Dziembowski, S., Maurer, U.: On generating the initial key in the bounded-storage model. In: Cachin, C., Camenisch, J. (eds.) EUROCRYPT 2004. LNCS, vol. 3027, pp. 126-137. Springer, Heidelberg (2004), ftp://ftp.inf.ethz.ch/pub/crypto/ publications/DziMau04b.pdf

8. Fehr, S., Schaffner, C.: Composing quantum protocols in a classical environment. In: Reingold, O. (ed.) TCC 2009. LNCS, vol. 5444, pp. 350-367. Springer, Heidelberg (2009)

${ }^{11}$ Well-formedness describes certain technical restrictions stemming from the proof by Ishai et al. 10: Whenever the functionality gets an input, the adversary is informed about the length of that input. Whenever the functionality makes an output, the adversary is informed about the length of that output and may decide when this output is to be scheduled. 
9. Håstad, J., Impagliazzo, R., Levin, L.A., Luby, M.: A pseudorandom generator from any one-way function. SIAM Journal on Computing 28(4), 1364-1396 (1999), full version http://www.ics . berkeley.edu/ luby/PAPERS/hill.ps

10. Ishai, Y., Prabhakaran, M., Sahai, A.: Founding cryptography on oblivious transfer - efficiently. In: Wagner, D. (ed.) CRYPTO 2008. LNCS, vol. 5157, pp. 572-591. Springer, Heidelberg (2008), http://www.spr.ingerlink.com/content/ 015v11524816u652/

11. Kilian, J.: Founding cryptography on oblivious transfer. In: STOC 1988, pp. 20-31. ACM, New York (1988)

12. König, R.,Wehner, S.,Wullschleger, J.: Unconditional security from noisy quantum storage. arXiv:0906.1030v2 [quant-ph] (June 2009)

13. Mayers, D.: Unconditionally Secure Quantum Bit Commitment is Impossible. Physical Review Letters 78(17), 3414-3417 (1997), http://arxiv.org/abs/quant-ph/ 9605044

14. Unruh, D.: Simulatable security for quantum protocols (September 2004), http://arxiv.o:g/ps/quant-ph/0409125

15. Unruh, D.: Universally composable quantum multi-party computation. In: Gilbert, H. (ed.) EUROCRYPT 2010. LNCS, vol. 6110, pp. 486-505. Springer, Heidelberg (2010), preprint on arXiv:0910.2912 [quant-ph]

16. Unruh, D.: Concurrent composition in the bounded quantum storage model. IACR ePrint 2010/229 (February 2011), full version of this paper

17. Wehner, S., Wullschleger, J.: Composable security in the bounded-quantum-storage model. In: Aceto, L., Damgård, I., Goldberg, L.A., Halldórsson, M.M., Ingólfsdóttir, A., Walukiewicz, I. (eds.) ICALP 2008, Part II. LNCS, vol. 5126, pp. 604-615. Springer, Heidelberg (2008), http://arxiv.0:g/abs/0709.0492v1

18. Wolf, S., Wullschleger, J.: Oblivious transfer is symmetric. In: Vaudenay, S. (ed.) EUROCRYPT 2006. LNCS, vol. 4004, pp. 222-232. Springer, Heidelberg (2006)

19. Wullschleger, J.: Oblivious-Transfer Amplification. Ph.D. thesis, ETH Zurich (March 2007), arXiv:cs/0608076v3 [cs.CR] 